ARTICLE

https://doi.org/10.1038/s41467-019-10983-7

\title{
Essentiality of sterol synthesis genes in the planctomycete bacterium Gemmata obscuriglobus
}

\author{
Elena Rivas-Marin (10) 1,4, Sean Stettner (1) 2,4, Ekaterina Y. Gottshall ${ }^{2,4}$, Carlos Santana-Molina (1) 1, \\ Mitch Helling (1) ${ }^{3}$, Franco Basile ${ }^{3}$, Naomi L. Ward ${ }^{2} \&$ Damien P. Devos (iD ${ }^{1}$
}

Sterols and hopanoids are chemically and structurally related lipids mostly found in eukaryotic and bacterial cell membranes. Few bacterial species have been reported to produce sterols and this anomaly had originally been ascribed to lateral gene transfer (LGT) from eukaryotes. In addition, the functions of sterols in these bacteria are unknown and the functional overlap between sterols and hopanoids is still unclear. Gemmata obscuriglobus is a bacterium from the Planctomycetes phylum that synthesizes sterols, in contrast to its hopanoid-producing relatives. Here we show that sterols are essential for growth of $G$. obscuriglobus, and that sterol depletion leads to aberrant membrane structures and defects in budding cell division. This report of sterol essentiality in a prokaryotic species advances our understanding of sterol distribution and function, and provides a foundation to pursue fundamental questions in evolutionary cell biology.

\footnotetext{
${ }^{1}$ Centro Andaluz de Biología del Desarrollo (CABD)-CSIC, Pablo de Olavide University, Seville 41013, Spain. ${ }^{2}$ Department of Molecular Biology, University of Wyoming, Laramie, WY 82071-2000, USA. ${ }^{3}$ Department of Chemistry, University of Wyoming, Laramie, WY 82071-2000, USA. ${ }^{4}$ These authors contributed equally: Elena Rivas-Marin, Sean Stettner, Ekaterina Y. Gottshall. Correspondence and requests for materials should be addressed to N.L.W. (email: nlward@uwyo.edu) or to D.P.D. (email: damienpdevos@gmail.com)
} 
S terols perform multiple essential functions in eukaryotic cell membranes, such as modulating membrane fluidity and permeability ${ }^{1-3}$, and allowing the formation of liquidordered membrane states (lipid rafts) ${ }^{4-6}$, which are critical for cell biology and pathogenesis. Sterol-induced membrane changes are also at the core of eukaryotic cell division, and thus, the emergence of sterols has long been recognized as a critical step in the development of the eukaryotes ${ }^{7}$.

Hopanoids and sterols are biosynthetically and functionally related polycyclic triterpenoids ${ }^{8}$. Most bacteria rely on hopanoid lipids to realize functions performed by eukaryotic sterols but their functional overlap is still unclear. However, a few bacteria produce sterols 9 . The function of bacterial sterols is currently unknown and the occurrence of sterol synthesis genes in bacteria has mostly been attributed to lateral gene transfer (LGT), either from eukaryotes to bacteria, or between bacteria ${ }^{9-11}$. This conundrum is exacerbated by the unclear evolutionary origins of both eukaryotic and bacterial sterol synthesis, despite the former's key role in eukaryogenesis, and the importance of sterols for geochemical dating ${ }^{12}$, where their presence is usually interpreted as evidence of eukaryotic life.

The role of sterols in bacteria is understudied. Sterol function has been investigated in the myxobacteria Stigmatella aurantiaca $^{13}$, which produces cycloartenol, a sterol mostly synthesized by plants. An insertion mutant of the cycloartenol synthase gene of $S$. aurantiaca was compared to the wild-type strain by transmission electron microscopy, and neither growth nor membrane structure phenotypes were detected. No mutant phenotypes were observed for other characters, such as ethanol sensitivity, swarming, or aggregation. It was thus concluded that sterol production is dispensable for growth in S. aurantiaca, a proteobacteria $^{13}$.

In order to broaden the studies of sterol function in bacteria, we experimentally investigated the contribution of sterol synthesis in Gemmata obscuriglobus, a member of the phylum Planctomycetes, unrelated to Proteobacteria. Further reasons to select G. obscuriglobus include that it possesses the most minimal sterol synthesis pathway yet reported, producing only lanosterol and its isomer parkeol ${ }^{14}$. In addition, G. obscuriglobus is the only planctomycete reported to produce sterols while all its relatives are potentially hopanoid producers. Also, the majority of planctomycetes have a complex endomembrane system, although it is arguably most developed in this species ${ }^{15}$. In eukaryotes, the membrane composition of sterols contributes to the function and organization of the cell's endomembrane system ${ }^{16}$. Thus, G. obscuriglobus is ideally suited to study the functional differences between hopanoids and sterols. Here, we address these fundamental questions through a combination of genetic, chemical, and microscopy approaches.

\section{Results}

Genetic interruption of sterol synthesis. In order to determine the phenotypic effects of sterol synthesis inhibition in G. obscuriglobus, we first applied a genetic approach. Sterols are synthesized from squalene by the enzymes squalene monooxygenase (Sqmo) and oxidosqualene cyclase (Osc). We independently interrupted the sqmo and osc genes, which are colocated in the G. obscuriglobus genome ${ }^{14}$ (Supplementary Figs. 1 and 2). Wild-type and mutant cells interrupted in non-essential genes form colonies on solid media after approximately 10 days of growth. Colonies were observed for the sqmo and osc mutants only after nearly a month. These colonies were of a more intense red color than the wild-type ones (Supplementary Fig. 3). Transconjugants were unable to grow when they were transferred to fresh solid medium or liquid broth, even in the presence of exogenous lanosterol. Thus, the mutant cells are unable to sustain normal unlimited growth. We speculate that the sterol-depleted colonies were able to undergo a few cycles of division, although inefficiently, by dilution of residual sterols present in their membranes. In order to demonstrate that the observed phenotype was due to the lack of sterols and not to plasmid integration, a similar plasmid containing a fragment of the sqmo gene under its own promoter was transferred into the wild-type strain. This chromosomal integration resulted in reconstruction of the wildtype operon, alongside the disrupted version, and produced cells resembling wild-type cells (Supplementary Fig. 1). Colonies appeared following 10 days of incubation, and in contrast to the interrupted mutants, these transconjugants could be regrown in liquid and solid medium, demonstrating that the osc or sqmo gene interruption was responsible for the originally observed nogrowth phenotype.

Chemical inhibition of squalene monooxygenase. We next applied a chemical approach that, in contrast to the genetic strategy, allowed partial inhibition of sterol synthesis, to permit further characterization of sterol depletion phenotypes. Terbinafine hydrochloride is an allylamine anti-fungal compound ${ }^{17}$ that specifically inhibits squalene epoxidase in fungi ${ }^{18}$. Based on sequence homology between G. obscuriglobus and eukaryotic Sqmo proteins ${ }^{14}$, we predicted that terbinafine hydrochloride could specifically inhibit the G. obscuriglobus Sqmo enzyme. Terbinafine treatment indeed reduced G. obscuriglobus growth in a dose-dependent manner (Fig. 1a), and a reduction in total sterol levels was observed (Fig. 1b), suggesting that the growth defect was specifically due to sterol biosynthesis inhibition. Supplementation of terbinafine-treated cultures with exogenous lanosterol substantially improved growth at all terbinafine doses (Fig. 1c), supporting our conclusions. Further evidence of sterol essentiality was provided by an experiment in which growth was completely suppressed by treatment with zaragozic acid, an inhibitor of squalene synthase ${ }^{19}$, but again rescued with the provision of exogenous lanosterol (Supplementary Fig. 4). This growth rescue is consistent with that observed in the terbinafine experiment (Fig. 1c). Lipotoxicity of accumulated squalene has been reported in yeast deficient in lipid storage organelles ${ }^{20}$, and a similar effect cannot be completely excluded for G. obscuriglobus at this time. However, the results of this experiment suggest that it is primarily sterol essentiality rather than squalene lipotoxicity that is responsible for the phenotypes observed in terbinafinetreated cultures and the interruption mutants.

Electron microscopy of sterol deficient cells. As sterols have never been reported as essential for bacterial growth, we attempted to identify phenotypic defects associated with their inhibition in G. obscuriglobus. Because of the lack of growth of the mutants, we investigated these defects by electron microscopy. In the sqmo mutant, we observed diverse striking phenotypes that have never been described in wild-type cells (Fig. 2 and Supplementary Fig. 5). A series of abnormal membrane conformations were observed, including nested vesicles, stacks of membrane layers inside the cytoplasm, multiple elongated sausage-like structures, cellular space crowded with small vesicles, or a mixture of these features (Fig. 2). We also observed numerous extracellular membranous structures, presumably cellular debris (Supplementary Fig. 5). Similar but milder phenotypes were also observed in terbinafine-treated cells (Supplementary Fig. 6). This supports the conclusion that the sqmo mutant phenotypes are indeed due to sterol depletion and not to the use of aged cellular material, necessitated by the slower growth of mutants. We also observed mutant cells that appear to be undergoing aberrant cell 

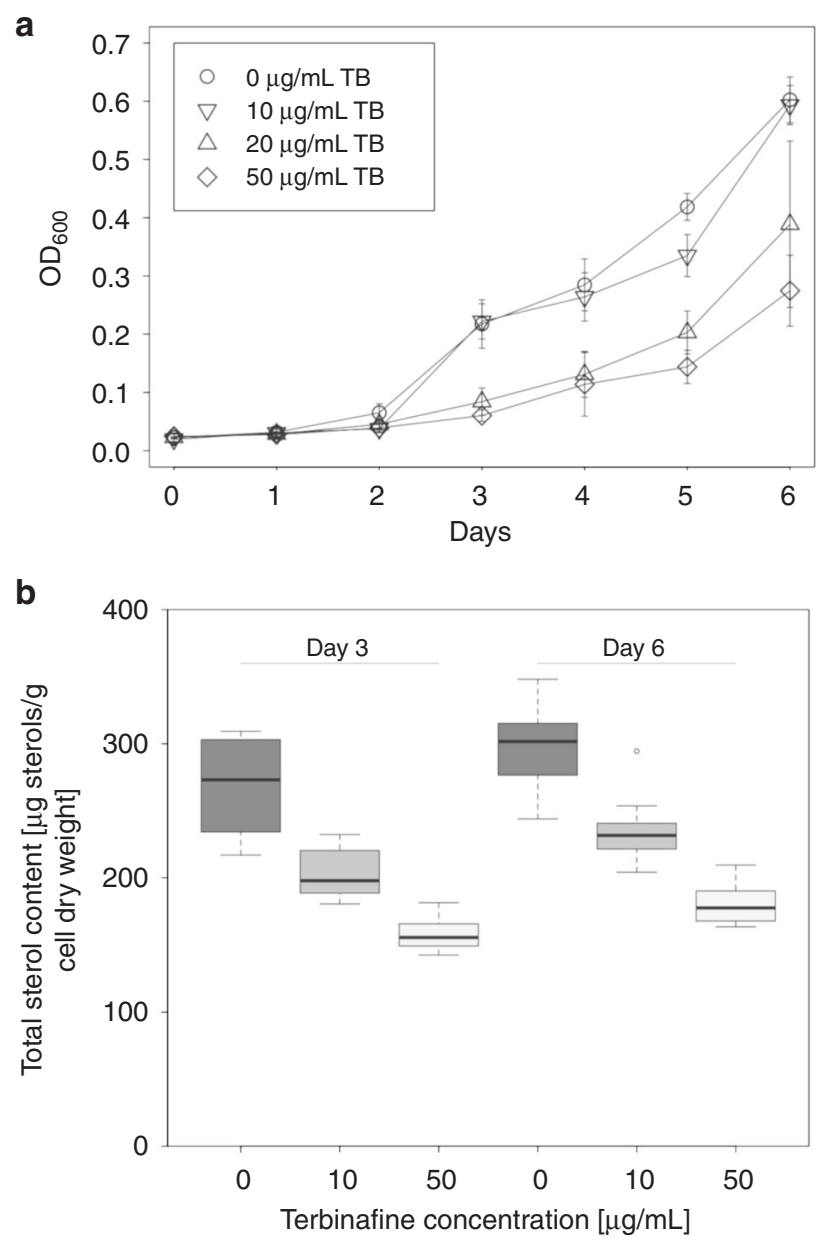

C

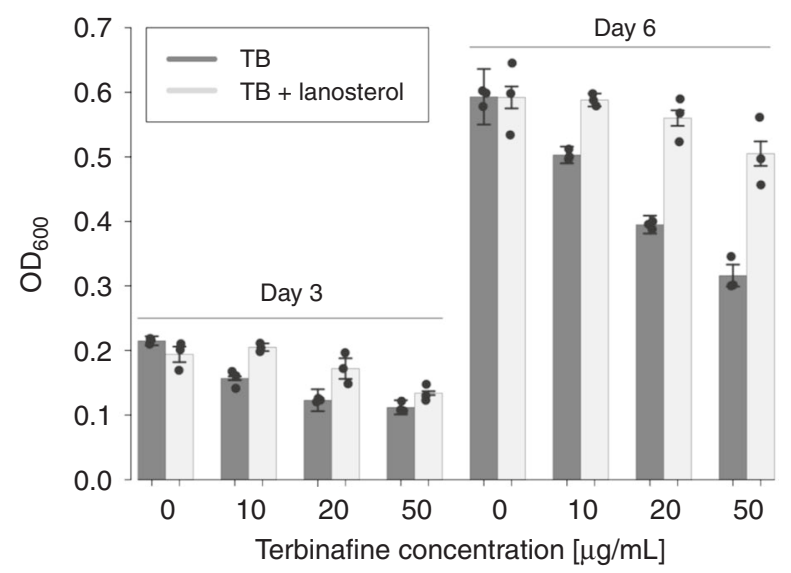

division, including cells with multiple buds and closed neck between cells (Fig. 2g, h). In contrast to wild-type dividing cells, the membranes at the bud of the necks in mutant cells seem to be occluding the length of the neck (Fig. 2a, g, h).

Time-lapse microscopy of inhibited cells. In order to observe these cellular defects in real-time, we returned to the chemical inhibition approach, monitoring terbinafine-treated cultures through time-lapse microscopy assays (Fig. 3; Supplementary Movies 1 and 2). Terbinafine addition resulted in the development of phase-bright inclusions in approximately one-third of the cells within $4-5 \mathrm{~h}$ after the start of the time-lapse experiment (Fig. $3 \mathrm{~b}-\mathrm{d}$ ), which roughly correspond to half a cell cycle in
Fig. 1 Terbinafine treatment suppresses growth and sterol production in Gemmata obscuriglobus, in a concentration-dependent manner. a Terbinafine (TB) treatment at various concentrations inhibits G. obscuriglobus growth relative to untreated cultures, measured spectrophotometrically over a 6-day time-course. Means and standard deviations are shown for three biological replicates. The points are represented by circles, upside-down triangles, triangles, and diamonds for increasing terbinafine concentrations, respectively. $\mathbf{b}$ Total cellular sterols, measured using gas chromatographymass spectrometry at 3-day and 6-day culture growth time points. Data is presented in boxplot of four biological replicates, each analyzed in triplicate. The center line represents the median, the box represents the upper and lower quartiles, and the lines represent the minimum and maximum observations. Any points outside the lines are considered outliers. c Lanosterol supplementation of TB-treated cultures partially or completely restores growth to the level of untreated cultures, measured spectrophotometrically at 3-day and 6-day culture growth time points. Means and standard deviations are shown for three biological replicates. Source data are provided as a Source Data file

G. obscuriglobus ${ }^{21}$. The cells containing inclusions appeared to be undergoing failed budding attempts, although we cannot exclude the possibility of dynamic membrane processes other than budding. Nevertheless, normal division was not observed in any cell containing an inclusion, and some of these cells appeared to prematurely release a phase-bright bud. The presence of inclusions was also associated with external cell debris, most likely related to that observed in the sqmo mutant (Supplementary Fig. 5). Addition of exogenous lanosterol led to the disappearance of these structures and to the rescue of budding replication (Fig. 3c, d and Supplementary Movies 1 and 2). This rescue effect strongly suggests that sterols are required for G. obscuriglobus cell division, the first reported evidence for an essential role of sterols in cell division outside of the domain Eukarya. To the best of our knowledge, hopanoids have never been reported to be essential for lab growth conditions nor have they been involved in cell division in bacteria, with one possible exception ${ }^{22}$. Because G. obscuriglobus is the only planctomycete reported to produce sterols, while all its relatives are hopanoid producers, the sterol essentiality reported here opens interesting avenues to study the functional differences between these molecules.

\section{Discussion}

We report the essentiality of bacterial sterol synthesis genes, at least under laboratory growth conditions. Our findings complement those of Gudde et al. ${ }^{23}$, who demonstrated in G. obscuriglobus that chemical inhibition of the downstream sterol synthesis enzyme, oxidosqualene cyclase, resulted in cell death. Although depletion of sterol levels was not directly detected, the authors achieved rescue of the growth defect with exogenous lanosterol supplementation, supporting the sterol inhibition as causative of cell death. Our study however provides the first evidence for an association between sterol synthesis, intracellular membrane morphology, and cell division, which is also novel for bacterial species. It appears likely that future studies of G. obscuriglobus, and perhaps other sterol-synthesizing bacteria, will identify novel functions of sterols in the context of bacterial cell biology. This will contribute to an improved understanding of fundamental similarities and differences between prokaryotic and eukaryotic cells.

\section{Methods}

Bacterial strains and culture conditions. The bacterial strains used in this work are summarized in Supplementary Table 1. Escherichia coli was grown in lysogeny broth (LB) medium at $37^{\circ} \mathrm{C}$, and G. obscuriglobus DSM $5831^{\mathrm{T}}$ strains in LB NaClfree medium $\mathrm{pH} 7.2$ at $28^{\circ} \mathrm{C}$ or PYGV medium (DSMZ 621) at $30^{\circ} \mathrm{C} .1 .5 \%$ bacto- 


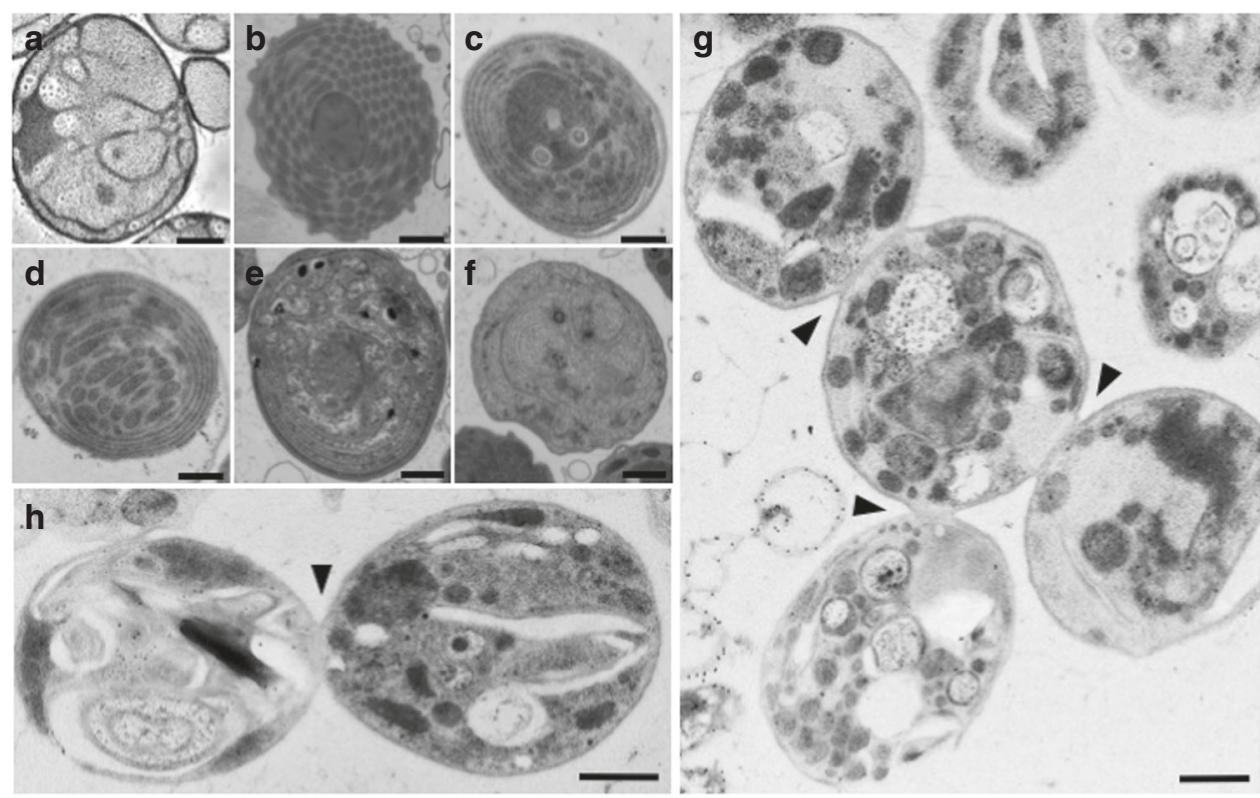

Fig. 2 Transmission electron microscopy of G. obscuriglobus cells deficient in sterol synthesis. Representative membrane phenotypes observed in the G. obscuriglobus mutant interrupted in the sqmo gene. Wild-type cell (a). Various membrane organizations are shown, including $\mathbf{b}$ cellular space crowded with small vesicles, $\mathbf{c}$ stacks of membrane layers inside the cytoplasm, $\mathbf{d}$ multiple elongated sausage-like structures, $\mathbf{e}, \mathbf{f}$ or a mixture of these and undefined features, $\mathbf{g}$ multiple budding. $\mathbf{g}$, h Arrows indicate necks between dividing cells. Black dots correspond to localization of the GFP used to interrupt the sqmo gene. Scale bar represents $0.5 \mu \mathrm{m}$
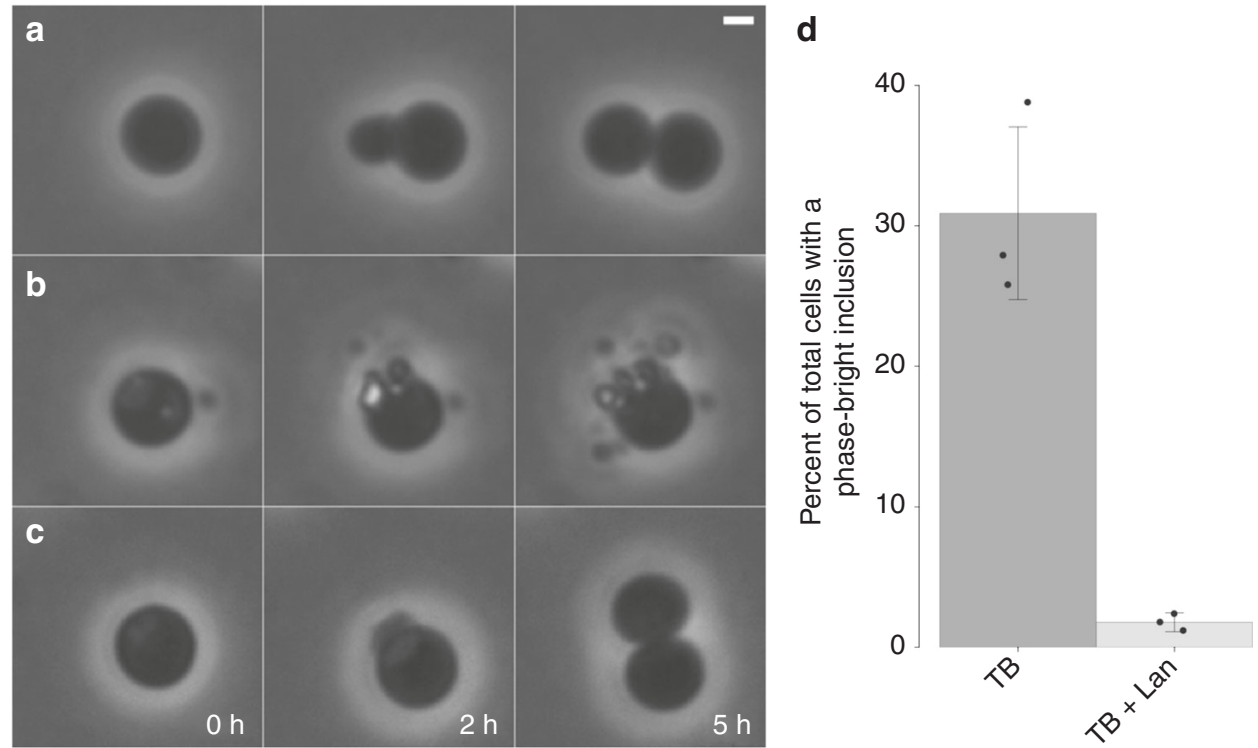

Fig. 3 Terbinafine treatment inhibits normal budding replication in Gemmata obscuriglobus. Selected frames of phase-contrast time-lapse microscopy of 4-day G. obscuriglobus cultures. a Control culture. b Culture treated with $100 \mu \mathrm{g} \mathrm{mL} \mathrm{L}^{-1}$ terbinafine. c Culture treated with $100 \mu \mathrm{g} \mathrm{mL} \mathrm{L}^{-1}$ terbinafine and $1 \mu \mathrm{g} \mathrm{mL} \mathrm{L}^{-1}$ lanosterol. a-c Scale bar represents $1 \mu \mathrm{m}$. d Incidence of phase-bright inclusions in TB-treated culture with or without lanosterol supplementation. Phasebright inclusions were never observed in untreated cultures. Means and standard deviations are shown for three biological replicates. Source data are provided as a Source Data file

agar was added for solid media. To avoid contamination of G. obscuriglobus cultures, $50 \mu \mathrm{g} \mathrm{mL}^{-1}$ cycloheximide (Cyclo) and $100 \mu \mathrm{g} \mathrm{mL}^{-1}$ ampicillin (Ap) were added. G. obscuriglobus cultures were grown aerobically in flasks, in a volume corresponding to one-fifth of a flask. When required, kanamycin $(\mathrm{Km})$ was used at the following concentrations $\left(\mu \mathrm{g} \mathrm{mL}^{-1}\right): 30$ for G. obscuriglobus and 25 for E. coli.

For determining the effects of terbinafine on the growth of G. obscuriglobus, an exponential-phase culture was inoculated to fresh liquid medium containing up to $50 \mu \mathrm{g} \mathrm{mL}^{-1}$ terbinafine hydrochloride (dissolved in ethanol), or $100 \mu \mathrm{LL}^{-1}$ ethanol (negative control) and subsequently incubated for up to 6 days. For complementation with exogenous lanosterol, PYGV medium containing up to 50 $\mu \mathrm{g} \mathrm{mL}^{-1}$ terbinafine hydrochloride and/or up to $5 \mu \mathrm{g} \mathrm{mL}^{-1}$ lanosterol (dissolved in DMSO) was used. Optical density was measured every $24 \mathrm{~h}$ at wavelength of $600 \mathrm{~nm}$ (OD600). Each experiment contained between three and five biological replicates.

An exponential phase culture was inoculated to fresh medium at a 1:1000 dilution containing one of the following: $40 \mu \mathrm{mL}^{-1}$ ethanol (negative control), $20 \mu \mathrm{g} \mathrm{mL}^{-1}$ zaragozic acid (dissolved in ethanol), $5 \mu \mathrm{g} \mathrm{mL}^{-1}$ lanosterol (dissolved in DMSO), or both lanosterol and zaragozic acid at their respective concentrations. OD600 was measured every $24 \mathrm{~h}$. Each experiment contained three biological replicates. 
Plasmids. The oligonucleotides and plasmids used in this work are summarized in Supplementary Tables 2 and 3, respectively. All DNA manipulations were made using standard protocols. Plasmid pMPO1012 ${ }^{24}$ was used as template to generate one-event homologous recombination insertion mutants. This is a ColE1mobilizable plasmid, containing the mut3a-gfp gene encoding a Green Fluorescent Protein expressed under a heterologous promoter, and an nptII kanamycin resistance gene. In order to support homologous recombination, 1232 and $1054 \mathrm{bp}$ fragments sqmo or osc genes, respectively, were amplified using genomic DNA as a template, and cloned into the pMPO1012 vector as a HindIII or HindIII/Klenowdirected fragment. The pDV011 plasmid was constructed to interrupt the sqmo gene by single homologous recombination using the primers Int SQMO Gemmata fwd and Int SQMO Gemmata rv (1232 bp). The pDV058 vector was built to disrupt the osc gene by single homologous recombination using the primers Int OSC Gemmata fwd and Int OSC Gemmata rv (1054 bp). Use of the pDV037 vector allowed interruption of the sqmo gene as achieved with pDV011, using the primers Int SQMO Gemmata 2 fwd and Int SQMO Gemmata rv (1511 bp), but resulting in regeneration of the full sterol synthesis operon after genome integration. The constructions are shown in Supplementary Fig. 1.

Genetic modification: triparental mating. Genetic transformation of G. obscuriglobus was performed by triparental mating using $200 \mu \mathrm{L}$ of an exponentially growing culture (OD600 0.4) of the donor (Supplementary Table 2) and helper (E. coli DH5a pRK2013) strains in LB, with a cell pellet from $15 \mathrm{~mL}$ of the receptor strain culture (OD600 0.4). Each culture was previously individually washed in phosphate buffer and resuspended in a total volume of $100 \mu \mathrm{L}$ of phosphate buffer that was spotted onto the corresponding agar plates containing Cyclo. The conjugation patches were incubated for $24 \mathrm{~h}$ at $28^{\circ} \mathrm{C}$, and resuspended in $1 \mathrm{~mL}$ of phosphate buffer. Transconjugants were plated on $\mathrm{Ap} 100 \mu \mathrm{g} \mathrm{mL}^{-1}$ and $\mathrm{Km} 30 \mu \mathrm{gL}^{-1}$ with or without lanosterol dissolved in chloroform $\left(20 \mu \mathrm{g} \mathrm{mL}^{-1}\right.$ from a $1000 \times$ stock). Viable cells were plated on Ap $100 \mu \mathrm{g} \mathrm{mL}^{-1}$. Colonies appeared after 10-30 days of incubation, depending on the plasmid integrated.

Characterization of modified strains by Southern blotting. Strains harboring the mutations was verified by fluorescence microscopy. Southern blotting analysis with $2 \mu \mathrm{g}$ of genomic DNA was also performed for the complemented strain (DV026). Genomic DNA was extracted using the Wizard Genomic DNA Purification Kit (Promega). The digested DNA was resolved by agarose gel electrophoresis. PCR amplicon used as probe was synthetized with the primers GFP pDV020 fwd and GFP pDV020 rv (Supplementary Table 1). Probes were labeled according to the manufacturer's instructions (DIG DNA Labeling Kit, Roche). DIG-labeled probes were detected with anti-Digoxigenin-AP, Fab fragments and CSPD (Roche). Visualizations were performed using Chemidoc XRS, and images were analyzed with the ImageLab 5.0 software.

Sterol extraction and derivatization. G. obscuriglobus pre-grown for 4 days in PYGV medium was used to inoculate $300 \mathrm{~mL}$ of fresh medium, supplemented with final concentrations of up to $50 \mu \mathrm{g} \mathrm{mL}^{-1}$ terbinafine and/or up to $1 \mu \mathrm{g} \mathrm{mL} L^{-1}$ lanosterol. All cultures contained Ap and Cyclo to a final concentration of $100 \mu \mathrm{g} \mathrm{mL}^{-1}$ and $50 \mu \mathrm{g} \mathrm{mL}^{-1}$, respectively. Two separate sets of cultures were grown for incubation periods of 3 days and 6 days. After incubation, pelleted cells were washed three times with phosphate-buffered saline, one time with $\mathrm{EtOH}$ to remove residual terbinafine, and finally one time with $\mathrm{ddH}_{2} \mathrm{O}$ to remove salts. All washes were performed with reagents at $4{ }^{\circ} \mathrm{C}$. Pellets were flash frozen in liquid nitrogen and lyophilized for $24 \mathrm{~h}$ (Freezone 6 Freeze Dry System Labconco). Dried pellets were kept at $-20^{\circ} \mathrm{C}$.

Dried pellets were weighed into $10 \mathrm{~mL}$ glass vessels (CEM Corp.) to obtain the dry weight of cell material. A $2 \mathrm{~mL}$ aliquot of 18 -megaohm deionized water was added to each vessel. Samples were spiked with $40 \mu \mathrm{L}$ of $120 \mu \mathrm{g} \mathrm{mL}-1$ D6lanosterol internal standard (Avanti Polar Lipids) in dichloromethane (DCM, Fisher Chemical). The solutions were sonicated by three 15-s, $6 \mathrm{~W}$ root mean squared (RMS) pulses while chilled on ice for cell lysis (Fisher Scientific 60 Sonic Dismembrator). The $\mathrm{pH}$ of each vessel was adjusted by the addition of $2 \mathrm{~mL}$ of $6 \%$ $(\mathrm{w} / \mathrm{w})$ potassium hydroxide in methanol. Vessels were sealed with crimp top caps (CEM Corp.), saponified at $90^{\circ} \mathrm{C}$ for $1 \mathrm{~h}$ in an oven, and then cooled to room temperature (RT). A $2 \mathrm{~mL}$ aliquot of DCM was added through the septa, vessels were vortexed for $5 \mathrm{~min}$ and then centrifuged for $3 \mathrm{~min}$ to separate the aqueous and organic layers. Sterols were extracted with DCM two times by suction of the organic layer with a glass pipette and extracts were combined in a new sample vial (Supelco 27149). The DCM solvent was evaporated in vacuum at RT. Extracts were resuspended in $100 \mu \mathrm{L}$ DCM and transferred to gas chromatography (GC) autosampler (AS) vials with $250 \mu \mathrm{L}$ inserts (Restek). A second $100 \mu \mathrm{L}$ DCM wash was used to rinse the sample vial and added to the GC AS vial for a more complete transfer. The DCM solvent was evaporated in vacuum at RT.

Derivatization of the dried extracts was performed by adding $40 \mu \mathrm{L}$ of MSTFA (N-methyl-N-trimethylsilyltrifluoroacetamide; Sigma Aldrich) to the GC AS vial inserts, capping the vials, and heating at $40^{\circ} \mathrm{C}$ for $30 \mathrm{~min}$ in an oven. The resulting solutions were directly analyzed by gas chromatography-mass spectrometry (GCMS). A series of lanosterol standard solutions ranging from 50 to $450 \mu \mathrm{g} \mathrm{mL}^{-1}$ were prepared, each with $120 \mu \mathrm{gL}^{-1}$ D6-lanosterol internal standard and derivatized with MSTFA as described above.
Gas chromatograph mass spectrometry and quantification. Sterol quantification was performed using a gas chromatograph (Trace GC Ultra) equipped with an autosampler (Triplus AutoSampler, liquid injection) and interfaced with a single quadrupole mass spectrometer (DSQ II; all from Thermo Scientific). GC instrument settings were: splitless injection, $1 \mu \mathrm{L}$ liquid injection volume, $220^{\circ} \mathrm{C}$ injector temperature. The oven program was: $150^{\circ} \mathrm{C}$ for $2 \mathrm{~min}, 20^{\circ} \mathrm{C} \mathrm{min}^{-1}$ to $330^{\circ} \mathrm{C}$, hold $3 \mathrm{~min}$. The transfer line temperature was held at $300^{\circ} \mathrm{C}$. Helium carrier gas was used at constant flow of $1.0 \mathrm{~mL} \mathrm{~min}^{-1}$. The column used was a (5\% phenyl)-95\% dimethylsiloxane (Phenomonex ZB-5 $30 \mathrm{~m} \times 0.25 \mathrm{~mm} \times 0.25 \mu \mathrm{m}$ ). MS settings were: positive ion mode with electron ionization, $70 \mathrm{eV}$ electron energy, $200 \mu \mathrm{A}$ filament emission current, $130 \mathrm{~V}$ lens, $250^{\circ} \mathrm{C}$ source temperature, the quadrupole scanned $\mathrm{m} / \mathrm{z} 50-650$ with a scan time of $0.35 \mathrm{~s}$. Samples, blanks, controls, and standards were randomized for injection order and injected in triplicate.

Lanosterol and parkeol quantification was performed with an internal standard (ISTD) multipoint calibration curve of lanosterol and D6-lanosterol as the ISTD. Peak area ratios of sterol $(\mathrm{m} / z$ 393) to ISTD $(\mathrm{m} / z$ 399) were measured in all samples and sterol content was computed from the calibration curve. Sterol content was then normalized to dry cell mass. For calculation of total sterol content, measurements for both sterols were summed. After testing for variance equivalency ( $F$-test), the twotailed Student's $t$-test at the $95 \%$ confidence interval was used to determine statistical support for differences in sterol content between different samples.

Electron microscopy. G. obscuriglobus mutant cells were scraped from the agar plates. The cells were frozen in an HPM010 (Abra Fluid, Switzerland) highpressure freezing machine and freeze substituted with either $1 \%$ osmium tetroxide, $0.1 \%$ uranyl acetate, and $5 \% \mathrm{H}_{2} \mathrm{O}$ and embedded in Epon or with $0.5 \%$ uranyl acetate and embedded in Lowicryl HM20. Thin sections were placed on formvarcoated grids and post-stained with uranyl acetate and lead citrate. Thin sections were imaged on a CM120 Phillips electron microscope ${ }^{25}$ For terbinafine inhibition assays, G. obscuriglobus cultures were grown with or without terbinafine at 10, 20, or $50 \mu \mathrm{g} \mathrm{mL}^{-1}$ for 4 days as described above. Cryofixation and freeze substitution were realized similarly and performed at the Molecular, Cellular, and Developmental Biology Electron Microscopy Facility, University of Colorado-Boulder. Ultrastructure images were acquired with use of a transmission electron microscope (Hitachi H-7000) at $75 \mathrm{kV}$, Gatan digital camera and Gatan software.

Phase-contrast time-lapse microscopy. G. obscuriglobus cells were grown for 4 days at $28^{\circ} \mathrm{C}$ in PYGV liquid medium with $100 \mu \mathrm{g} \mathrm{mL}^{-1}$ of terbinafine or without. $1 \mathrm{~mL}$ of each culture was pelleted, washed once in phosphate-buffered saline, and resuspended in sterile $\mathrm{ddH}_{2} \mathrm{O}$. This suspension was spotted onto a $1 \%$ agarose-PYGV pad on a glass slide. The pad contained $100 \mu \mathrm{g} \mathrm{mL}^{-1}$ terbinafine for the treated cells. The pad was covered with a glass coverslip and sealed with a mixture of equal amount of petroleum jelly, lanolin, and paraffin wax (VALAP), leaving enough empty space around the agarose pad to provide the cells with sufficient oxygen. A single field of cells was imaged every 30 min during $14 \mathrm{~h}$ on a Zeiss Axio Imager Z2 epifluorescence microscope equipped with a Hamamatsu Orca-Flash4.0 sCMOS camera and a Plan-Apochromat 100×/1.46 Oil Ph3 objective. The images were collected in the Zen Blue software and processed using Image $\mathrm{J}$ and the EBImage package in Program $\mathrm{R}^{26}$.

Reporting summary. Further information on research design is available in the Nature Research Reporting Summary linked to this article.

\section{Data availability}

The authors declare that all other data supporting the findings of this study are available within the paper and its Supplementary Information files, or from the corresponding author on request. Source data are provided as a Source Data file.

Received: 10 January 2018 Accepted: 10 June 2019

Published online: 02 July 2019

\section{References}

1. London, E. Insights into lipid raft structure and formation from experiments in model membranes. Curr. Opin. Struct. Biol. 12, 480-486 (2002).

2. Demel, R. A. \& De Kruyff, B. The function of sterols in membranes. Biochim. Biophys. Acta 457, 109-132 (1976).

3. Tyler, K. M. et al. Flagellar membrane localization via association with lipid rafts. J. Cell Sci. 122, 859-866 (2009).

4. Anderson, R. G. W. \& Jacobson, K. A role for lipid shells in targeting proteins to caveolae, rafts, and other lipid domains. Science 296, 1821-1825 (2002).

5. Brown, D. A. \& London, E. Structure and function of sphingolipid- and cholesterol-rich membrane rafts. J. Biol. Chem. 275, 17221-17224 (2000).

6. Jacobson, K. \& Dietrich, C. Looking at lipid rafts? Trends Cell Biol. 9, 87-91 (1999). 
7. Cavalier-Smith, T. The phagotrophic origin of eukaryotes and phylogenetic classification of Protozoa. Int. J. Syst. Evol. Microbiol. 52, 297-354 (2002).

8. Sáenz, J. P. et al. Hopanoids as functional analogues of cholesterol in bacterial membranes. Proc. Natl Acad. Sci. USA 112, 11971-11976 (2015).

9. Wei, J. H., Yin, X. \& Welander, P. V. Sterol synthesis in diverse bacteria. Front. Microbiol. 7, 990 (2016).

10. Chen, L.-L., Wang, G.-Z. \& Zhang, H.-Y. Sterol biosynthesis and prokaryotesto-eukaryotes evolution. Biochem. Biophys. Res. Commun. 363, 885-888 (2007).

11. Desmond, E. \& Gribaldo, S. Phylogenomics of sterol synthesis: insights into the origin, evolution, and diversity of a key eukaryotic feature. Genome Biol. Evol. 1, 364-381 (2009).

12. Summons, R. E. \& Lincoln, S. A. Biomarkers: informative molecules for studies in geobiology. In Fundamentals of Geobiology (eds Knoll, A. H. et al.) 269-296 (Blackwell Publishing Ltd., 2012).

13. Bode, H. B. et al. Steroid biosynthesis in prokaryotes: identification of myxobacterial steroids and cloning of the first bacterial 2,3(S)-oxidosqualene cyclase from the myxobacterium Stigmatella aurantiaca. Mol. Microbiol. 47, 471-481 (2003).

14. Pearson, A., Budin, M. \& Brocks, J. J. Phylogenetic and biochemical evidence for sterol synthesis in the bacterium Gemmata obscuriglobus. Proc. Natl Acad. Sci. USA 100, 15352-15357 (2003).

15. Devos, D. P. PVC bacteria: variation of, but not exception to, the Gramnegative cell plan. Trends Microbiol. 22, 14-20 (2014).

16. Bacia, K., Schwille, P. \& Kurzchalia, T. Sterol structure determines the separation of phases and the curvature of the liquid-ordered phase in model membranes. Proc. Natl Acad. Sci. USA 102, 3272-3277 (2005).

17. Petranyi, G., Meingassner, J. G. \& Mieth, H. Antifungal activity of the allylamine derivative terbinafine in vitro. Antimicrob. Agents Chemother. 31, 1365-1368 (1987).

18. Ryder, N. S. Mechanism of action and biochemical selectivity of allylamine antimycotic agents. Ann. N. Y. Acad. Sci. 544, 208-220 (1988).

19. Bergstrom, J. D. et al. Zaragozic acids: a family of fungal metabolites that are picomolar competitive inhibitors of squalene synthase. Proc. Natl Acad. Sci. USA 90, 80-84 (1993).

20. Valachovic, M., Garaiova, M., Holic, R. \& Hapala, I. Squalene is lipotoxic to yeast cells defective in lipid droplet biogenesis. Biochem. Biophys. Res. Commun. 469, 1123-1128 (2016).

21. Lee, K.-C., Webb, R. \& Fuerst, J. The cell cycle of the planctomycete Gemmata obscuriglobus with respect to cell compartmentalization. BMC Cell Biol. 10, 4 (2009).

22. Doughty, D. M. et al. The RND-family transporter, HpnN, is required for hopanoid localization to the outer membrane of Rhodopseudomonas palustris TIE-1. Proc. Natl Acad. Sci. USA 108, E1045-E1051 (2011).

23. Gudde, L. R., Hulce, M., Largen, A. H. \& Franke, J. D. Sterol synthesis is essential for viability in the planctomycete bacterium Gemmata obscuriglobus. FEMS Microbiol. Lett. 366, fnz019 (2019).

24. Rivas-Marín, E., Canosa, I., Santero, E. \& Devos, D. P. Development of genetic tools for the manipulation of the planctomycetes. Front. Microbiol. 7, 914 (2016).

25. Santarella-Mellwig, R., Pruggnaller, S., Roos, N., Mattaj, I. W. \& Devos, D. P. Three-dimensional reconstruction of bacteria with a complex endomembrane system. PLoS Biol. 11, e1001565 (2013).
26. Pau, G., Fuchs, F., Sklyar, O., Boutros, M. \& Huber, W. EBImage-an R package for image processing with applications to cellular phenotypes. Bioinformatics 26, 979-981 (2010).

\section{Acknowledgements}

N.L.W. thanks Dr. Grant Bowman for use of microscopy equipment, and Dr. Ann Pearson for helpful discussions in the early stages of this project. E.R.-M., C.S.-M., and D. P.D. were supported by BFU2013-40866-P and BFU-2016-7826-P. S.S., E.Y.G., M.H., F B., and N.L.W. were supported by the US National Science Foundation awards MCB0920667 and IOS-1656637. S.S. and E.Y.G. were additionally supported by the US National Institutes of Health awards NCRR-P20RR016474 and NIGMS-P20GM103432. The acquisition of the GC-MS was made possible by the US National Science Foundation award CHE-0844694 to F.B.

\section{Author contributions}

All authors designed the experiments. E.R.-M., S.S., E.Y.G., C.S.-M., and M.H. performed the experiments and analyzed the data. E.R.-M., S.S., E.Y.G., M.H., F.B., N.L.W., and D.P. D. wrote the manuscript. All authors reviewed and edited the manuscript. All authors read and approved the final manuscript.

\section{Additional information}

Supplementary Information accompanies this paper at https://doi.org/10.1038/s41467019-10983-7.

Competing interests: The authors declare no competing interests.

Reprints and permission information is available online at http://npg.nature.com/ reprintsandpermissions/

Peer review information: Nature Communications thanks Paula Welander and other anonymous reviewer(s) for their contribution to the peer review of this work. Peer reviewer reports are available.

Publisher's note: Springer Nature remains neutral with regard to jurisdictional claims in published maps and institutional affiliations.

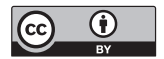

Open Access This article is licensed under a Creative Commons Attribution 4.0 International License, which permits use, sharing, adaptation, distribution and reproduction in any medium or format, as long as you give appropriate credit to the original author(s) and the source, provide a link to the Creative Commons license, and indicate if changes were made. The images or other third party material in this article are included in the article's Creative Commons license, unless indicated otherwise in a credit line to the material. If material is not included in the article's Creative Commons license and your intended use is not permitted by statutory regulation or exceeds the permitted use, you will need to obtain permission directly from the copyright holder. To view a copy of this license, visit http://creativecommons.org/ licenses/by/4.0/.

(c) The Author(s) 2019 